\title{
Clinical outcome of sacral neuromodulation in incomplete spinal cord injured patients suffering from neurogenic lower urinary tract symptoms
}

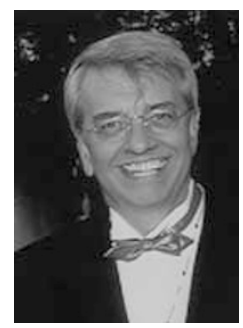

\author{
JJ Wyndaele, Editor \\ Antwerp University Hospital, Antwerp, Belgium \\ E-mail: spinalcord@uza.be
}

Dear Spinal Cord reader,

Two indications for neuromodulation are clearly valid in urology: urinary incontinence (for overactive bladder syndrome: OABS) and chronic urinary retention. It still remains uncertain how neuromodulation works, but regardless of the hypothesis, most researchers agree that the somatic afferents are the vectors, as the visceral nerve fibers cannot be directly activated by the stimulation intensities normally used today with this technique. This implies that neuromodulation cannot be effective in patients with a non-functional peripheral nerve circuit. Patients with incomplete medullar lesions, whether of traumatic or other origins, may benefit from neuromodulation, as shown in previous studies with a very small number of patients. On the other hand, all researchers agree that patients with complete medullar lesions would not benefit from neuromodulation. Lombardi and Del Popolo present the largest study so far, using 24 incomplete SCI patients, half of whom have urinary retention and half of whom have OABS. With a median follow-up of 61 months, a clinical improvement of more than $50 \%$ compared with baseline could be found. Side effects and need for re-operation are described.

There are two reviews in this issue. Silver and Weiner review the treatment of spinal deformity in 19th-century Europe and explain the high incidence of paralysis as a result of forceful traction of the spinal column to treat scoliosis in France, as compared with other European countries. Although well described in 19th-century French medical literature, the dangers of forceful traction upon the spinal column to correct spinal deformity were not recognised in Europe or the USA until the halo-femoral traction method of treatment was used in the 1970s. This provides a good example of how long it can take before medical information reaches those most involved.

Noonan et al. critically reviewed six instruments that assess participation in persons with spinal cord injury. They conclude that it is important to determine what information about participation is required before selecting an instrument and give good examples of such choice.

Munce et al. present an interesting survey on physician utilization by individuals with traumatic SCI. They demonstrate significant physician utilization, especially among family physicians and physiatrists. While the factors predicting higher physician and specialist utilization may reflect individuals with the most severe impairment, co-morbid conditions or lack of social support, the model for higher emergency department visits may point to limited accessibility to/availability of primary care services for SCI patients in rural regions.

We continue to publish additional data on clinical results of cellular therapy for chronic spinalcord-injured patients. Moviglia et al. present a case series in which a combination of three cell therapies and an appropriate neurorehabilitation program were used to recreate and enhance the natural conditions that SCI repairs. In the following issues more studies on the same topic will appear.

These are just some examples of the interesting articles published in this June issue of Spinal Cord. You will also find several other high-quality contributions.

Spinal Cord (2009) 47, 427; doi:10.1038/sc.2009.53 